\title{
Adaptive modeling of the femtosecond inscription in silica
}

\section{Vladimir Mezentsev, Jovana Petrovic, Jürgen Dreher, Rainer Grauer}

Vladimir Mezentsev, Jovana Petrovic, Jürgen Dreher, Rainer Grauer, "Adaptive modeling of the femtosecond inscription in silica," Proc. SPIE 6107, Laser-based Micropackaging, 61070R (23 February 2006); doi: $10.1117 / 12.647303$

Event: Lasers and Applications in Science and Engineering, 2006, San Jose, California, United States 


\title{
Adaptive modeling of the femtosecond inscription in silica
}

\author{
Vladimir Mezentsev ${ }^{a}$, Jovana Petrovic ${ }^{a}$, Jürgen Dreher $^{b}$, and Rainer Grauer ${ }^{b}$ \\ ${ }^{a}$ Photonics Research Group, Aston University, Birmingham B4 7ET, United Kingdom; \\ ${ }^{b}$ Theoretische Physik I, Ruhr-Universität Bochum, D-44780 Bochum, Germany
}

\begin{abstract}
We present an adaptive mesh approach to high performance comprehensive investigation of dynamics of light and plasma pattens during the process of direct laser inscription. The results reveal extreme variations of spatial and temporal scales and tremendous complexity of these patterns which was not feasible to study previously.
\end{abstract}

Keywords: Self-focusing, femtosecond inscription, adaptive mesh refinement

\section{INTRODUCTION}

Direct inscription of the complex microstructures in refractive materials by means of intense femtosecond radiation is one of the novel enabling technologies in modern photonics. This technology implies that pre-focused femtosecond light pulses produce phase transitions and create domains with a modified refractive index. Nonlinear propagation of femtosecond intense laser pulses in dielectrics exhibits a wide range of fascinating phenomena including conical emission, ${ }^{1}$ X-waves,${ }^{2}$ self-reconstruction, ${ }^{3}$ self-healing ${ }^{2}$ light filaments plasma breakdown. ${ }^{4}$ Proof of principle experiments of the potential of fs inscription for microfabrication of photonic structures was demonstrated almost a decade ago. ${ }^{5}$ It has now become a very promising method of microfabrication. ${ }^{6-8}$ Other interesting applications aim to achieve the longest possible self-guiding filaments to deliver the energy through the bulk of material ${ }^{9}$

Self-focusing of the intense laser pulse is a key physical phenomenon leading to a multi-photon ionization at its final stage. In fact the very formation of plasma filaments limits the catastrophic damage due to defocusing and multi-photon absorption. Eventually, the thermalization and recombination of the plasma filament leads to the modification of medium and a distributed profile of refractive index is produced. The dynamics of the light-induced plasma filaments is extremely complex and defined by many factors. It is an extremely fast process evolving at the very fine spatial scales. In this paper, we have developed and adaptive numerical approach to the detailed study of the evolution of plasma filaments and the role of pulse and media parameters on the shape of resulting filaments. We consider a geometry in which an incident Gaussian input field is focused through a lens into the planar silica sample sample. For the purposes of this paper, only one focusing configuration and a single initial pulsewidth are considered. To demonstrate the principle of adaptive mesh approach, we use a simplified version of the widely accepted model of the nonlinear propagation of the laser pulse as it was formulated in Ref.. ${ }^{10}$ It is essentially a nonlinear Schrödinger equation (NLSE) coupled with an equation describing the plasma generation. This basic model describes effects of self-focusing, multiphoton absorption (MPA), and group velocity dispersion (GVD). Pure NLSE is a generic model which ultimately appears in consistent description of envelope amplitude of the nonlinear wave packets. It is widely used to describe fs light propagation ${ }^{11}$ and has been extensively studied. One of the most striking features of the NLSE is catastrophic self-focusing or beam collapse which means a formation of a singularity in finite propagation length. ${ }^{12}$ Beam collapse happens in the framework stationary NLSE if the pulse power exceeds a certain critical value. Formally, the on-axis intensity achieves an infinitely high value at some critical propagation length. However, in the extended physical models which account for dispersion and nonlinear absorption a formation of singularity is arrested. ${ }^{13}$ The nonlinear evolution of the collapsing beam with the presence of the arresting effects is extremely rich. Mathematically it poses a stiff multidimensional evolution problem. Straightforward numerical modeling of such problems is a very

Further author information: (Send correspondence to V.M.)

V.M.: E-mail: v.mezentsev@aston.ac.uk, Telephone: +44 1212043481

Laser-based Micropackaging, edited by Friedrich G. Bachmann, Willem Hoving, Yongfeng Lu, Kunihiko Washio, Proc. of SPIE Vol. 6107, 61070R, (2006) · 0277-786X/06/\$15 - doi: 10.1117/12.647303 


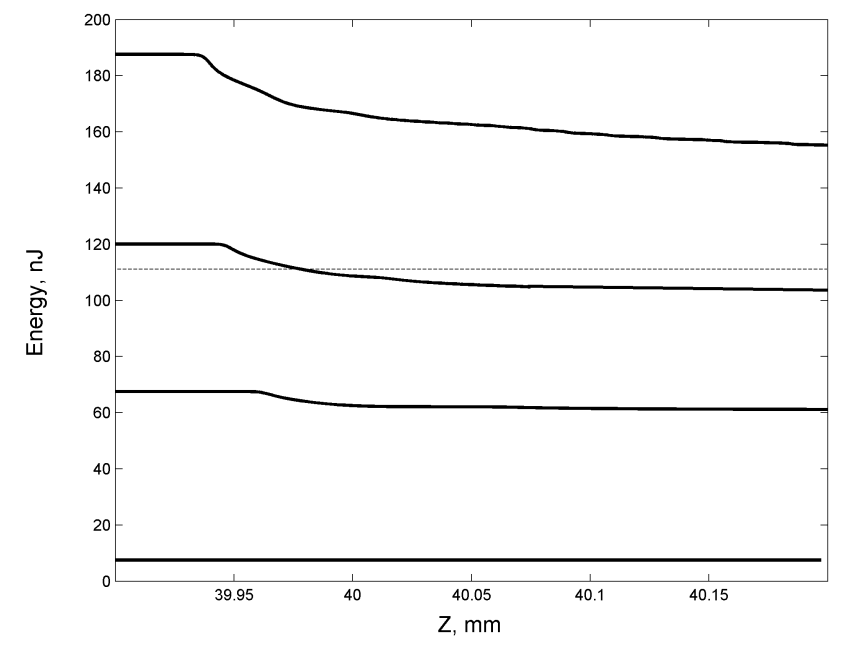

Figure 1. Pulse energy as a function of the propagation distance for different input peak powers. Horizontal dashed line corresponds to the critical power for a given initial pulsewidth $t_{0}=60 \mathrm{fs}$.

difficult challenge due to the multiscale nature of underlying physical phenomena. In this paper, we address an intrinsic stiffness of the mathematical problem by introducing a hierarchy of the adaptively refined grids which are dynamically adjusted for proper resolution of the fine spatial structures and temporal features of the beam. We report on the development of a portable computational framework for the parallel, mesh-adaptive solution of system of a 3D parabolic wave equation for envelope amplitude of electromagnetic field coupled with the rate equation for plasma density. Local mesh refinement is realized by the recursive bisection of grid blocks along each spatial and temporal dimensions. Implemented numerical schemes include standard finite-difference and spectral methods. Non-adaptive solver has also been implemented for back-to-back accuracy tests and performance profiling. Parallel execution is achieved through a configurable hybrid of POSIX-multi-threading and MPI-distribution with dynamic load balancing.

\section{THEORETICAL MODEL}

\subsection{Equations}

This section describes a theoretical model used for femtosecond pulse propagation in silica. It was originally suggested by Feit and Fleck $^{14}$ and later developed into a fairly complex model, see e.g. Refs. ${ }^{9,15-21}$ For the purposes of this paper, a simplified model is used, essentially similar to that described by Feng et al. ${ }^{10}$ :

$$
\begin{gathered}
i \mathcal{E}_{z}+\frac{1}{2 k} \Delta_{\perp} \mathcal{E}-\frac{k^{\prime \prime}}{2} \frac{\partial^{2} \mathcal{E}}{\partial t^{2}}+k_{0} n_{2}|\mathcal{E}|^{2} \mathcal{E}=-\frac{i \sigma}{2}(1+i \omega \tau) \rho \mathcal{E}-i \frac{\beta^{(K)}}{2}|\mathcal{E}|^{2(K-1} \mathcal{E} \\
\frac{\partial \rho}{\partial t}=\frac{1}{n_{b}^{2}} \frac{\sigma_{b s}}{E_{g}} \rho|\mathcal{E}|^{2}+\frac{\beta^{(K)}}{K \hbar \omega}|\mathcal{E}|^{2 K}
\end{gathered}
$$

The terms on the left-hand side of Eq.(1) describe effects of beam diffraction, group velocity dispersion (GVD), and Kerr nonlinearity. The latter is responsible for a catastrophic self-focusing which is limited by the effects described by terms on the right-hand side of Eq.(1), namely plasma absorption and multi-photon absorption. In Eq.(1) the laser beam propagation along the $z$ axis is assumed and this equation is essentially a reduced paraxial approximation of the wave equation for the complex electric field envelope $\mathcal{E}$ with a carrier frequency $\omega$ in the moving frame of coordinates. Here $k=n_{b} k_{0}=n_{b} \omega / c$ is the propagation vector, $k^{\prime \prime}=\partial^{2} k(\omega) / \partial \omega^{2}$ is the GVD parameter, $n_{b}(\omega)$ is a linear refractive index of the bulk medium, $n_{2}$ is the nonlinear coefficient describing nonlinear self-modulation (Kerr effect) such that $n_{2}|\mathcal{E}|^{2}$ is a nonlinear contribution to the refractive 
index, $\sigma_{b s}$ is the cross section for inverse Bremsstrallung, $\tau$ is the electron relaxation time, $E_{g}$ is the ionization energy, and the quantity $\beta^{(K)}$ controls the $K$-photon absorption. Equation (2) implements the Drude model for electron-hole plasma in the bulk of silica and describes the evolution of the electron density $\rho$. The first term on the right-hand side is responsible for the avalanche impact ionization and the second term - for the ionization resulting from MPA. Equation (2) is suitable for description of the sub-picosecond laser pulses when plasma diffusion is negligible. Here, the wave equation describing the evolution of the focused optical beam in the form of NLSE (left-hand terms in Eq.(1)) which is extended to include plasma generation, pulse-plasma interaction, and MPA (terms on the right-hand of Eq.(1). Group velocity dispersion included in Eq.(1) has been shown to lead to pulse splitting and to arrest the collapse. ${ }^{13,22-26}$

\subsection{Physical parameters}

In all our simulations the Gaussian initial condition:

$$
\mathcal{E}(z=0, r, t)=\sqrt{\frac{2 P_{i n}}{\pi r_{0}^{2}}} \exp \left(-\frac{r^{2}}{r_{0}^{2}}-\frac{i k r^{2}}{2 f}-\frac{t^{2}}{t_{p}^{2}}\right),
$$

where $r_{0}$ is the waist of the incident beam, $t_{p}$ defines the conventionally defined pulsewidth $t_{F W H M}=\sqrt{2 \ln 2} t_{p} \approx$ $1.177 t_{p}$, and $f$ is a focal length of the objective lens.

For all our simulations a fixed single values of $r_{0}=2.5 \mathrm{~mm}$ and $t_{p}=60 \mathrm{fs}$ was used. This fixed pulsewidth corresponds to the critical energy of $116 \mathrm{~nJ}$ for a critical power $P_{c r}=\lambda_{0}^{2} / 2 \pi n_{b} n_{2} \approx 2.3 \mathrm{MW}$ in fused silica with $n_{b}=1.453$ being the linear refraction index and $n_{2}=3.2 \times 10^{-16} \mathrm{~cm}^{2} / \mathrm{W}$ the nonlinear refraction index. Critical power is proven to be a crucial parameter to determine the evolution of the collapsing beam. We assume the laser wavelength $\lambda_{0}$ to be $800 \mathrm{~nm}$ and the focusing lens to have $f=40 \mathrm{~mm}$.

The other parameters for fused silica, used in simulations are described below. GVD coefficient $k^{\prime \prime}=361$ $\mathrm{fs}^{2} / \mathrm{cm}$, inverse Bremsstrahlung cross section $\sigma_{b s}=2.78 \times 10^{-18} \mathrm{~cm}^{2}$. Multiphoton absorption coefficient can be expressed as $\beta^{(K)} \hbar \omega \sigma_{K} \rho_{a t}$, whith $\rho_{a t}=2.1 \times 10^{22}$ atoms $/ \mathrm{cm}^{3}$ being a material concentration and $\sigma_{K}=$ $1.3 \times 10-55 \mathrm{~cm}^{2 K} / \mathrm{W}^{K} / \mathrm{s}$. We assume five-photon ionization with $K=5$ and $E_{g}=7.6 \mathrm{eV}$ in fused silica.

The quation (2) can be expressed as

$$
\frac{\partial}{\partial t} \frac{\rho}{\rho_{B D}}=\frac{1}{n_{b}^{2}} \frac{\sigma_{b s}}{E_{g}} \frac{\rho}{\rho_{B D}}|\mathcal{E}|^{2}+|\mathcal{E}|^{2 K}
$$

where

$$
I_{M P A}=\left(\frac{K \hbar \omega \rho_{B D}}{\beta^{(K)}}\right)^{1 / K}
$$

is defined to be an MPA threshold as the plasma ionization rate becomes very steep when the intensity $I$ exceeds $I_{M P A} \cdot \rho_{B D}=1.7 \times 10^{21}$ is a plasma breakdown density. Being important physical thresholds, both $I_{M P A}$ and $\rho_{B D}$ were used in a very useful normalization of physical variables to dimensionless ones for performing simulations. The choice of normalization is irrelevant to discuss as all the parameters and fields throughout this paper are produced with their physical values.

\section{ADAPTIVE NUMERICAL METHOD}

The principle of adaptive mesh refinement is rather simple. Starting with one grid of given resolution (in most of our 3D configurations we currently chose $512 \times 64 \times 64$ mesh points) called master grid, the partial differential equations (1-2) are solved with a scheme summarized below. After a certain number of steps along propagation axis $z$, it is checked whether the local numerical resolution is still sufficient on the entire grid. If it is detected that finer grid is locally needed, a refinement is carried out and a child grid is created using the interface with a parent grid as a new boundary. In order to prepare for it, the points where the error of discretization exceeds a given value are marked on the grid. In addition to these grid points, adjacent ones are included. These marked points of insufficient numerical resolution have to be covered with rectangular grids of finer resolution as efficiently as possible. Our algorithm for this purpose is very similar to the one used by Berger and Colella, ${ }^{27}$ and it was 
$\rho / \rho_{B D}$

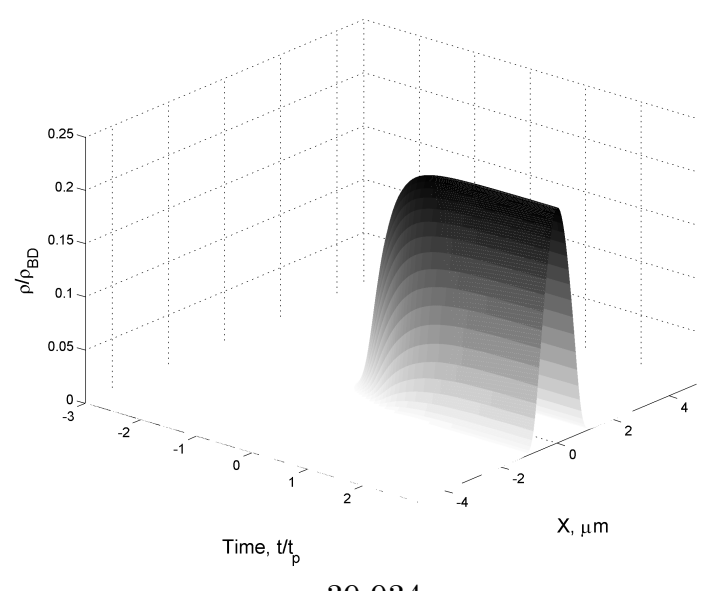

$z=39.934 \mathrm{~mm}$

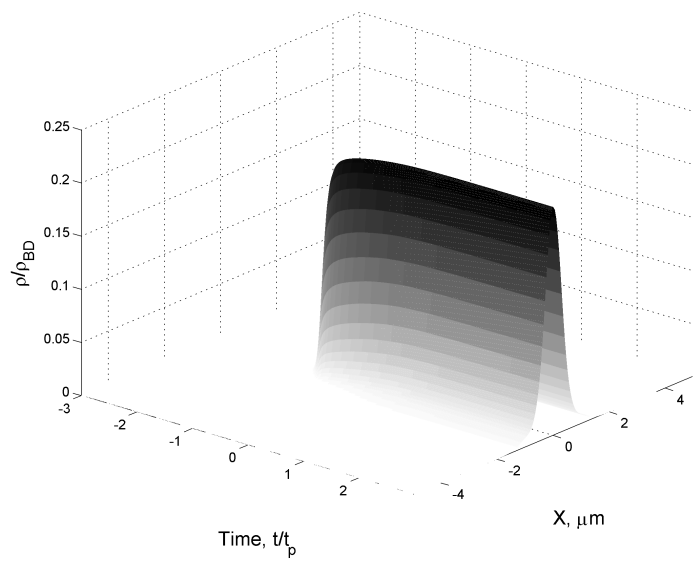

$z=39.953 \mathrm{~mm}$

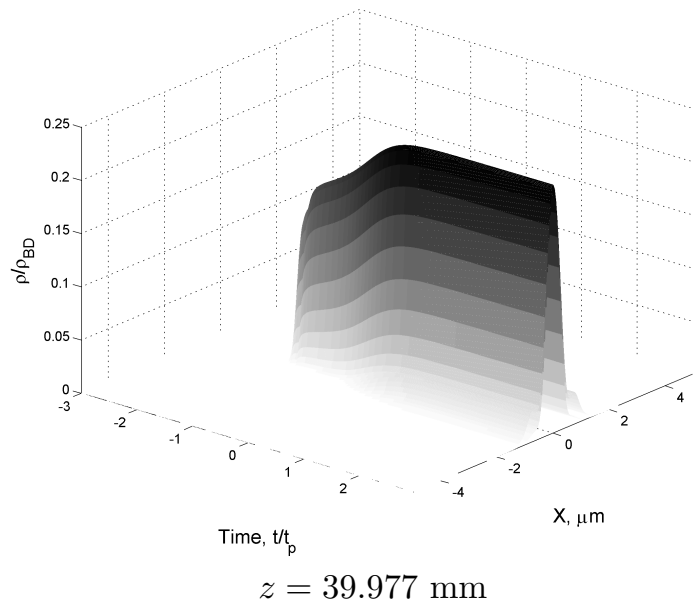

$z=39.977 \mathrm{~mm}$
$I / I_{M P A}$
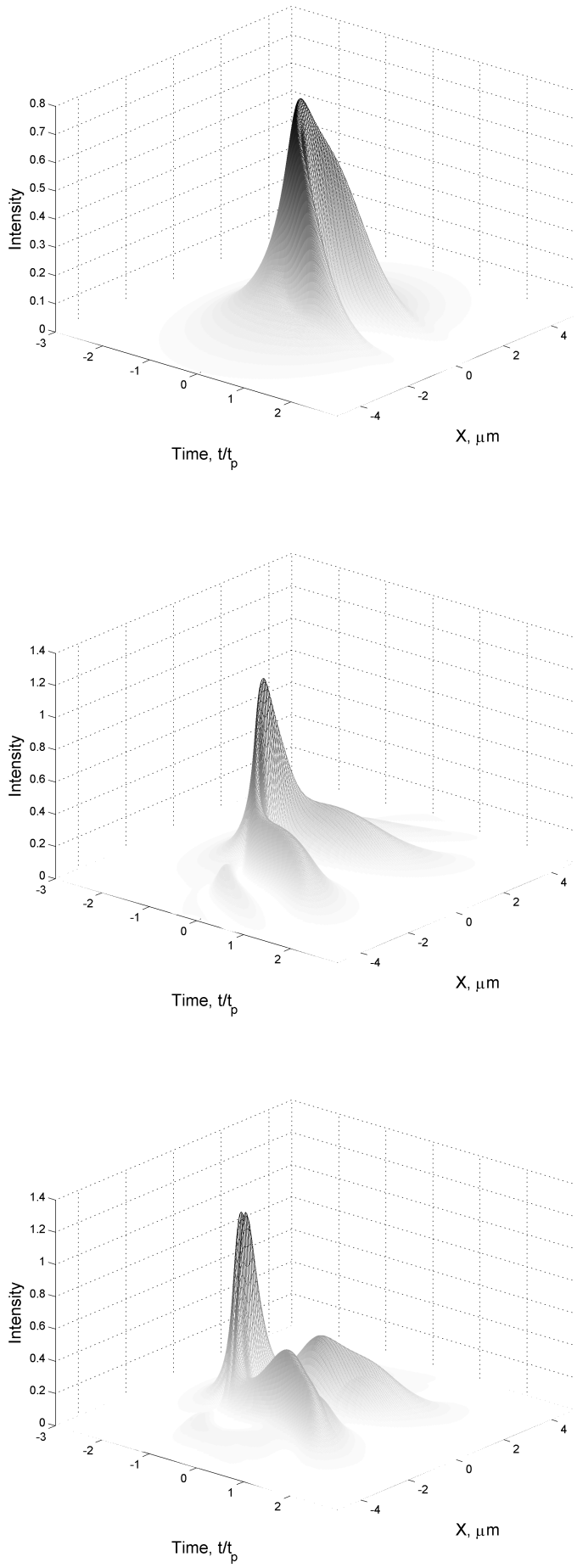

Continued ...

Figure 2. Dynamics of the intensity and the plasma density at different positions along axis $z$. 


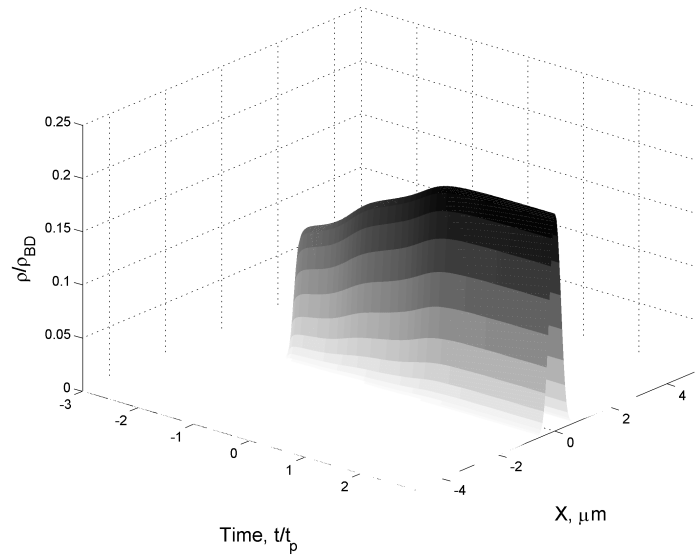

$z=39.983 \mathrm{~mm}$

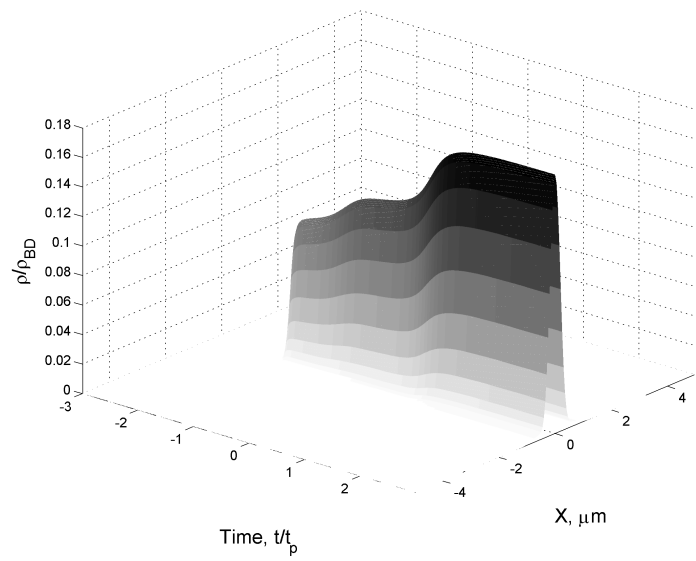

$z=39.991 \mathrm{~mm}$

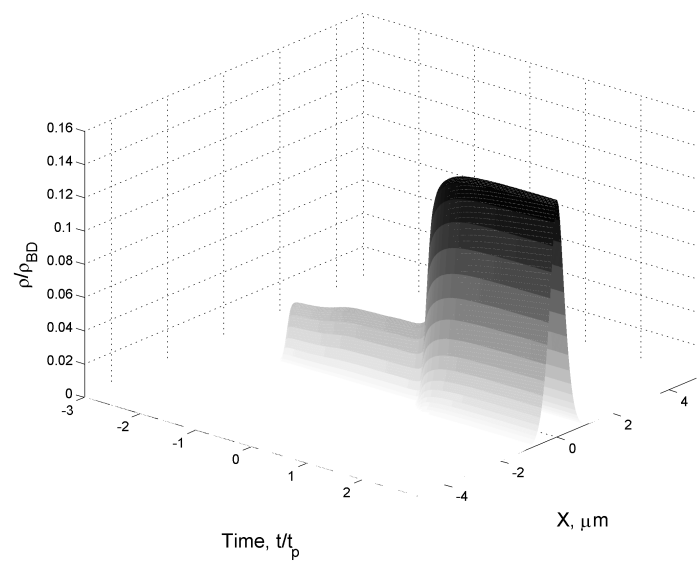

$z=40.005 \mathrm{~mm}$
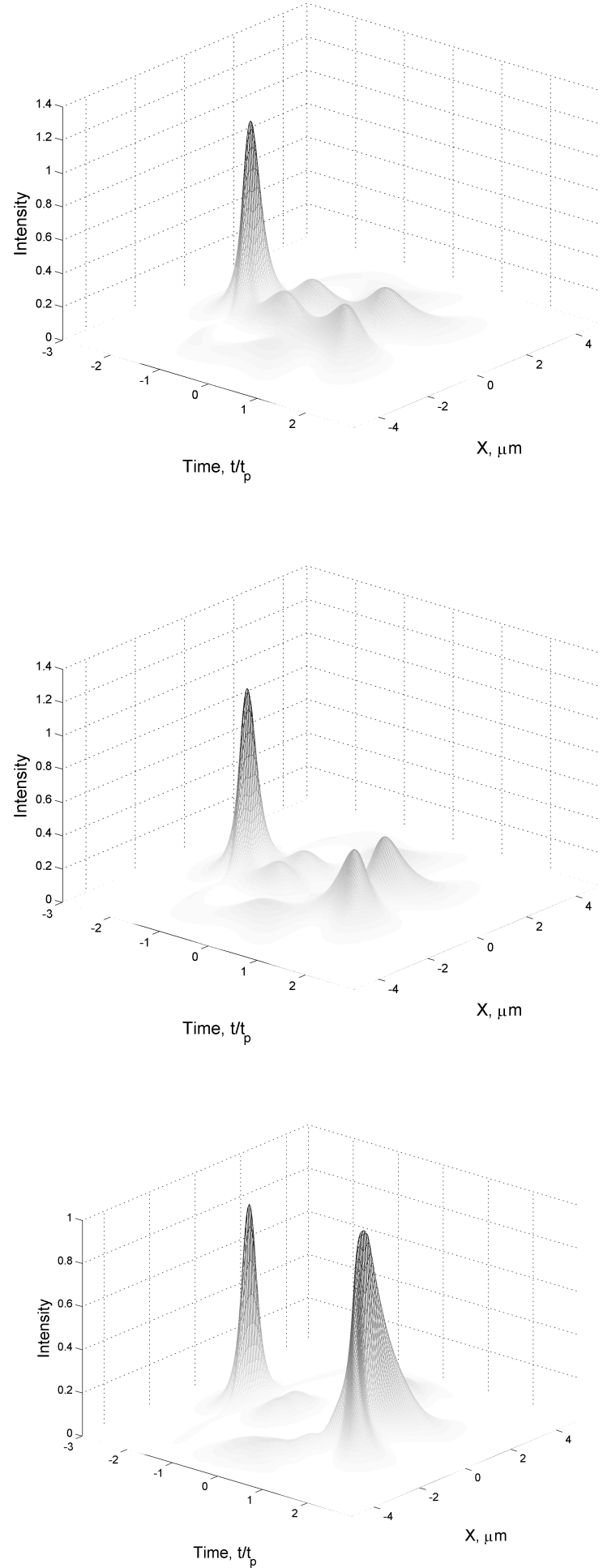

Figure 3. (Fig. 2 cont...)Dynamics of the intensity and the plasma density at different positions along axis $z$. 


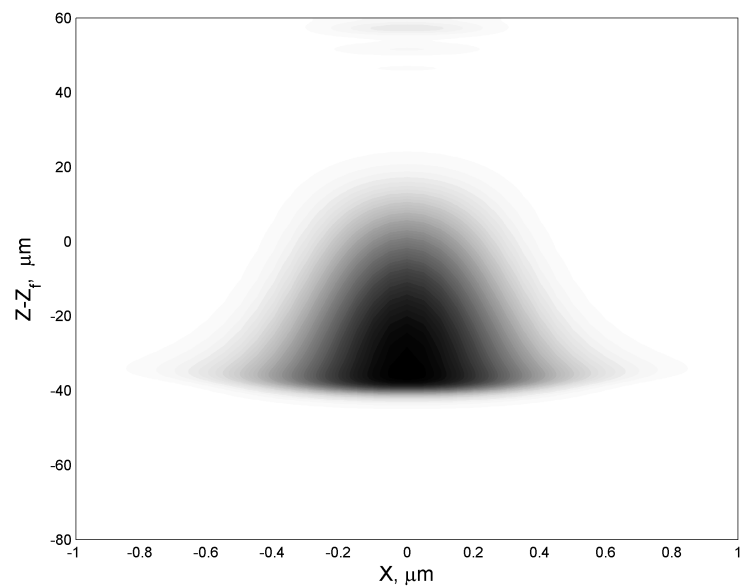

\section{Subcritical}

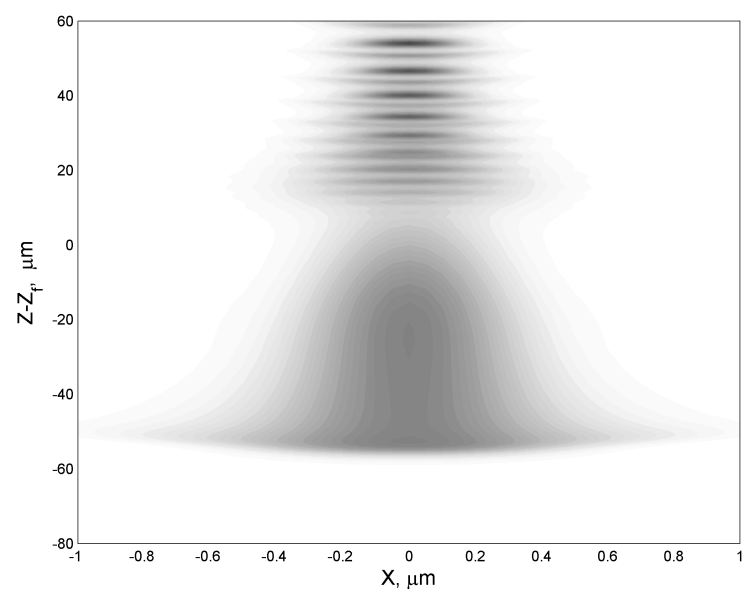

\section{Critical}

\section{Supercritical}

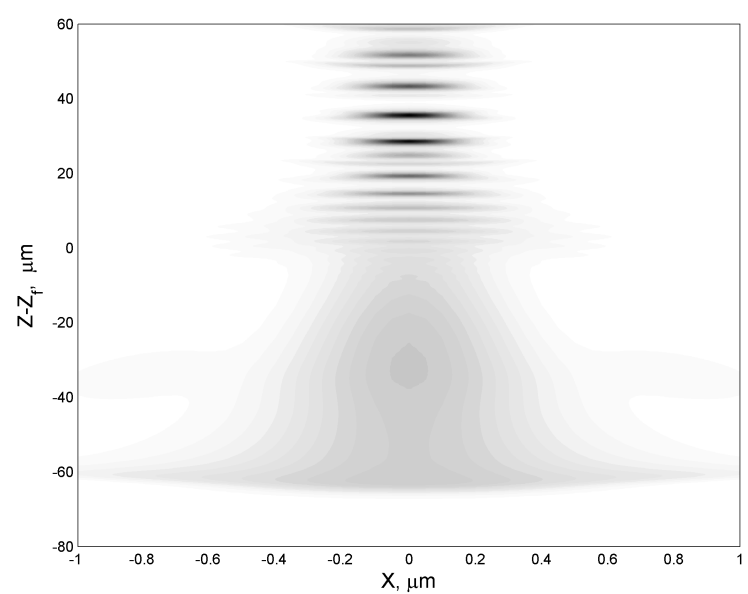

Figure 4. Contours of the plasma density at infinite time after electric field is vanished for different energies corresponding to the initial values in Fig.(1): Supercritical - $187 \mathrm{~nJ}$; Critical - 120 nJ, Subcritical - 68 nJ. 
described in detail by Friedel et al.. ${ }^{28}$ On the child grids, the spatial discretization length and the time step are reduced by a certain refinement factor. The new grids are filled with data obtained by interpolation from the preceding parent (coarser) level. The integration advances on both the parent and the child levels until the local resolution becomes insufficient again. The rebuilding of the grid hierarchy starting with the current level and proceeding on all subsequent levels begins when the above-mentioned threshold for the error is locally exceeded, e.g., if the domains of high intensity have left the finer grids, or if local gradients have developed, such that the prescribed accuracy is not guaranteed. The points of insufficient numerical resolution are collected on all grids of each level. On the basis of the resulting list of these points, new grids are generated. After assuring that the newly generated grids are properly embedded in their parent grids, interpolated data are filled in. If data existed on grids of the same level before the regridding, these are substituted to the interpolated data from the parents grids.

Solution of the model described in Introduction in the outlined framework requires selecting an appropriate numerical integration scheme. It turned out that the optimal performance can be achieved by using a different scheme on the refined levels than on the base level, when taking into account that arbitrary mesh sizes on the refined levels occurred. On the base level, we applied an operator-splitting method which is second-order accurate in $z$. Radial diffraction term in the Laplacian operator of Eq.(1) is integrated by means of Crank-Nicholson scheme with zero boundary condition at the maximum radius. Both the angular term of the transverse diffraction operator and the dispersion operator are diagonal in Fourier space thus Fast Fourier Transform was utilized for numerical integration of these terms. It is worth noting that neither periodic or zero global boundary conditions both in transverse space and time do not impose a significant restriction on the problem considered, because the localized wave-packets vanish at the boundaries during the entire process of focusing and defocusing after the pulse passes the focal domain. On the refined meshes we apply a semi-implicit scheme of Crank-Nicholson type, which was used by, e.g., Pietsch et al. ${ }^{29}$ and also utilized in a previous modelling on NLSE with normal dispersion. ${ }^{13}$ The proposed discretization reads

$$
\left[1-\frac{i \Delta z}{2}\left(L_{q l m}^{p}+\frac{i \Delta z}{2} \partial_{z}\left(p_{n l}\right)_{q l m}^{p}\right)\right]^{-1} u_{q l m}^{p+1}=\left[1+\frac{i \Delta z}{2}\left(L_{q l m}^{p}+\frac{i \Delta z}{2} \partial_{z}\left(p_{n l}\right)_{q l m}^{p}\right)\right]^{-1} u_{q l m}^{p}
$$

with $L=\kappa \Delta_{\perp}+\delta \partial_{t}^{2}+p_{n l}$ and

$$
p_{n l}=s|u|^{2}-i \frac{\gamma}{2}(1+i \omega \tau) \rho-i \mu|u|^{2 K}
$$

where $\kappa$, delta, $s, \nu$, and $\mu$ are dimensionless parameters introduced in an obvious way. To invert the operator on the left-hand side of Eq.(6), Helmholtz-type equation must be solved. Since this linear operator is close to identity for small steps $\Delta z$. A standard Gauss-Seidel relaxation method is employed with red/black ordering on each grid. In order to solve the problem globally, an additive Schwarz iteration ${ }^{30}$ is performed on top of the per-grid solvers. It is important to comment on the refinement criterion. We calculate the right-hand side of Eq.(6) based on the actual grid spacing and twice the grid spacing. When the difference exceeds a given threshold, those mesh points are marked under-resolved and are subject to refinement. The threshold value conditioning the refinement was determined such that a sufficient resolution was guaranteed during the evolution along the propagation axis $z$. The length of the integration step $\Delta z$ was dynamically adapted to ensure that at all times the i) Courant-Friedrich-Levy condition was met to enforce the numerical stability and the iterative method converged at a prescribed minimal rate and ii) the maximal relative increment of both the amplitude and the nonlinear phase resulting from Eq.(7) was always kept less than a prescribed limit, usually $1 \%$.

The implementation of the adaptive mesh refinement strategy described above is done in $\mathrm{C}++$. We make use of a non-adaptive solver described above for handling the master grid. This solver was also used for benchmarking and back-to-back accuracy testing of adaptive solver. Handling of the data structures is separated from the problem under consideration. Therefore, it is relatively easy to use the code for other types of problems including various generalizations of the system Eqs.(1-2). Since on each grid the advance along $z$ and the Helmholtz-type equation can be solved independently and the number of grids supersedes the number of processors available, parallelization is highly efficient. 


\section{NONLINEAR DYNAMICS}

There are two distinct typical setups related to the focusing geometry. If the goal is to produce the longest possible filament, then the loose focusing and small spot size are required. ${ }^{2,9,31}$ However in the microfabrication context the opposite goal is usually desirable. The focused spot is often required to be as small as possible and the absorbed energy is needed to be within a narrow window between the thresholds of inscription and damage. This is an extremely difficult challenge because of the huge difference in spatial and temporal scales of the incident laser beam and fine features of light and plasma patterns in the vicinity of the focal point. The adaptive procedure described above allows for accurate treatment of multiscale evolution which results in stationary (in the framework of the model considered) distribution of plasma. The mechanisms of eventual plasma recombination and subsequent relaxation of the medium are extremely complex. For example, the latter can be described as a sophisticated 3D thermo-elasto-plastic processes. ${ }^{32}$ The purpose of the present work is to find an accurate spatial distribution of plasma needed for such or similar subsequent analysis. Typical asymptotic plasma density profiles for different initial pulse energies are shown energies are shown in Figure 4. Density plots of plasma concentrations are shown in one transverse and one propagation coordinates. It shows that subcritical evolution $\left(P_{i n}<P_{c r}\right)$ leads to a smooth plasma cloud without visible fine structure. This regime is probably the most attractive from the microfabrication viewpoint because it is characterized by the smooth subcritical evolution of the peak intensity. Larger energies lead to development of the pronounced periodic fringes which result from the relaxation oscillations of a collapsing beam after the collapse is arrested by the multiphoton absorption.

Nontrivial complex light and plasma dynamics and formation of interesting light patterns are illustrated by Figures 2-3. Snapshots of the beam intensity profile in transverse space and time are presented at different points along the propagation axis in the vicinity of the focal point for supercritical case $P_{i n}>P_{c r}$. Originally, an intensity profile forms a distinct crescent in $x-t$ plane. It is then evolved so that the shoulders of this crescent split from the front of the pulse and form a pair of satellite pulses which rise being fed by a contracting beam. This pair is eventually coalesce to form a secondary crescent pulse following the remains of the front pulse. Then this scenario repeats for the newborn crescent pulse which even has the same amplitude just under the MPA threshold. A cascade of such crescents result in periodic fringes of plasma density until finally significant fraction of the original pulse energy is absorbed.

\section{CONCLUSION}

A high performance adaptive mesh platform is developed for comprehensive numerical modeling of the femtosecond inscription of photonic structures in silica during the process of nonlinear propagation of tightly focused femtosecond laser radiation. Proof of principle is demonstrated and a few cases studies is performed. Detailed numerical modeling of fine dynamics in the vicinity of the focal point reveals an extremely complex behavior of the focused light coupled to generated plasma. The resulting light and plasma patterns have very complex fine structure which was impossible to establish prior to this study.

\section{ACKNOWLEDGMENTS}

We acknowledge technical support of technical staff running Cray XD1 computing cluster facility in School of Engineering and Applied Science at Aston Univercity.

\section{REFERENCES}

1. O. G. Kosareva, V. P. Kandidov, A. Brodeur, C. Y. Chien, and S. L. Chin, "Conical emission from laserplasma interactions in the filamentation of powerful ultrashort laser pulses in air," Opt. Lett. 22, pp. 13321334, 1997.

2. M. Kolesik, E. M. Wright, and J. Moloney, "Dynamic nonlinear $\mathrm{x}$-waves for femtosecond pulse propagation inwater," Phys. Rev. Lett. 92, p. 253901, 2004.

3. A. Dubietis, E. Kucinskas, G. Tamosauskas, E. Gaizauskas, M. A. Porras, and P. D. Trapani, "Selfreconstruction of light filaments," Opt. Lett. 29, pp. 2893-2895, 2004. 
4. C. W. Carr, M. D. Feit, A. M. Rubenchik, P. D. Mange, S. O. Kucheyev, M. D. Shirk, H. B. Radousky, and S. G. Demos, "Radiation produced by femtosecond laserplasma interaction during dielectric breakdown," Opt. Lett. 30, pp. 661-663, 2005.

5. K. M. Davis, K. Miura, N. Sugimoto, and K. Hirao, "Writing waveguides in glass with a femtosecond laser," Opt. Letters 21, pp. 1729-1731, 1996.

6. C. B. Schaffer, A. Brodeur, J. F. Garcìa, and E. Mazur, "Micromachining bulk glass by use of femtosecond laser pulses with nanojoule energy," Opt. Lett. 26, pp. 93-95, 2001.

7. A. H. Nejadmalayeri, P. R. Herman, J. Burghoff, M. Will, S. Nolte, and A. Tuennermann, "Inscription of optical waveguides in crystalline silicon by mid-infrared femtosecond laser pulses," Opt. Lett. 30, pp. 964966, 2005.

8. A. M. Kowalevicz, V. Sharma, E. P. Ippen, J. G. Fujimoto, and K. Minoshima, "Three-dimensional photonic devices fabricated in glass by use of a femtosecond laser oscillator," Opt. Lett. 30, pp. 1060-1062, 2005.

9. S. Tzortzakis, L. Sudrie, M. Franco, B. Prade, A. Mysyrowicz, A. Couairon, and L. Bergé, "Self-guided propagation of ultrashort ir laser pulses in fused silica," Phys. Rev. Lett. 21, p. 213902, 2001.

10. Q. Feng, J. V. Moloney, A. C. Newell, E. M. Wright, K. Cook, P. K. Kennedy, D. X. Hammer, B. A. Rockwell, and C. R. Thompson, "Theory and simulation on the threshold of water breakdown induced by focused ultrashort laser pulses," IEEE Journal of Quantum Electronics 33, pp. 127-137, 1997.

11. J. H. Marburger, "Self-focusing: theory," Prog. Quantum Electron. 4, pp. 35-110, 1975.

12. V. P. S.N. Vlasov and V. Talanov Izv. Vuzov, Radiofizilca 14, pp. 1353-1364, 1971.

13. K. Germaschewski, R. Grauer, L. Bergé, V. K. Mezentsev, and J. J. Rasmussen, "Splittings, coalescence, bunch and snake patterns in the $3 \mathrm{~d}$ nonlinear schr"

14. M. D. Feit and J. A. Fleck, "Effect of refraction on spot-size dependence of laser-induced breakdown," Appl. Phys. Lett. 24, pp. 169-172, 1974.

15. T. Brabec and F. Krausz, "Nonlinear optical pulse propagation in the single-cycle regime," 78, pp. 32823285, 1997.

16. J. K. Ranka and A. L. Gaeta, "Breakdown of the slowly varying envelope approximation in the self-focusing of ultrashort pulses," Opt. Lett. 23, pp. 534-536, 1998.

17. A. L. Gaeta, "Catastrophic collapse of ultrashort pulses," Phys. Rev. Lett. 84, pp. 3582-3585, 2000.

18. M. Kolesik, J. V. Moloney, and M. Mlejnek, "Unidirectional optical pulse propagation equation," Phys. Rev. Lett. 89, p. 283902, 2002.

19. S. Tzortzakis, L. Bergé, M. Franco, B. Prade, A. Mysyrowicz, and A. Couairon, "Infrared femtosecond light filaments in air: simulations and experiments," J. Opt. Soc. Am. B 19, pp. 1117-1129, 2002.

20. M. Kolesik, G. Katona, J. Moloney, and E.M.Wright, "Physical factors limiting the spectral extent and band gap dependence of supercontinuum generation," Phys. Rev. Lett. 91, p. 043905, 2003.

21. R. Nuter, S. Skupin, and L. Bergé, "Chirp-induced dynamics of femtosecond filaments in air," Opt. Lett 30, pp. 917-919, 2005.

22. N. A. Zharova, A. G. Litvak, T. A. Petrova, A. M. Sergeev, and A. D. Yunakovskii, "Multiple fractionation of wave structures in a nonlinear medium," Pisma Zh. Eksp. Teor. Fiz. (JETP Lett.) 44, pp. 12-15, 1986.

23. P. Chernev and V. Petrov, "Self-focusing of light pulses in the presence of normal group velocity dispersion," Opt. Lett. 17, pp. 172-174, 1992.

24. J. E. Rothenberg, "Pulse splitting during self-focusing in normally dispersive media," Opt. Lett. 17, pp. 583$585,1992$.

25. G. G. Luther, J. V. Moloney, A. C. Newell, and E. M. Wright, "Selffocusing threshold in normally dispersive media," Opt. Lett. 19, pp. 862-864, 1994.

26. G. G. Luther, A. C. Newell, and J. V. Moloney, "The effect of normal dispersion on collapse events," Physica D 74, pp. 59-73, 1994.

27. M. J. Berger and P. Colella, "Local adaptive mesh refinement for shock hydrodynamics," J. Comp. Phys. 82, pp. 64-84, 1989.

28. H. Friedel, R. Grauer, and C. Marliani, "Adaptive mesh refinement for singular current sheets in incompressible magnetohydrodynamic flows," J. Comp. Phys. 134, pp. 190-198, 1997. 
29. H. Pietsch, E. Laedke, and K.-H. Spatschek, "Nonlinear mode conversion with chaotic soliton generation at plasma resonance," Phys. Rev. E 47, pp. 1977-1995, 1993.

30. W. Hackbusch, Iterative Solution of Large Sparse Systems of Equations, Springer, New York, 1994.

31. A. Dubietis, G. Tamoauskas, I. Diomin, and A. Varanaviiuset, "Self-guided propagation of femtosecond light pulses in water," Opt. Lett 28, pp. 1269-1271, 2003.

32. X. Zhang, X. Xu, and A. Rubenchik, "Simulation of microscale densification during femtosecond laser processing of dielectric materials," Appl. Phys. A 79, pp. 945-948, 2004. 